\section{Scottish practices invest in the latest scanning technology}

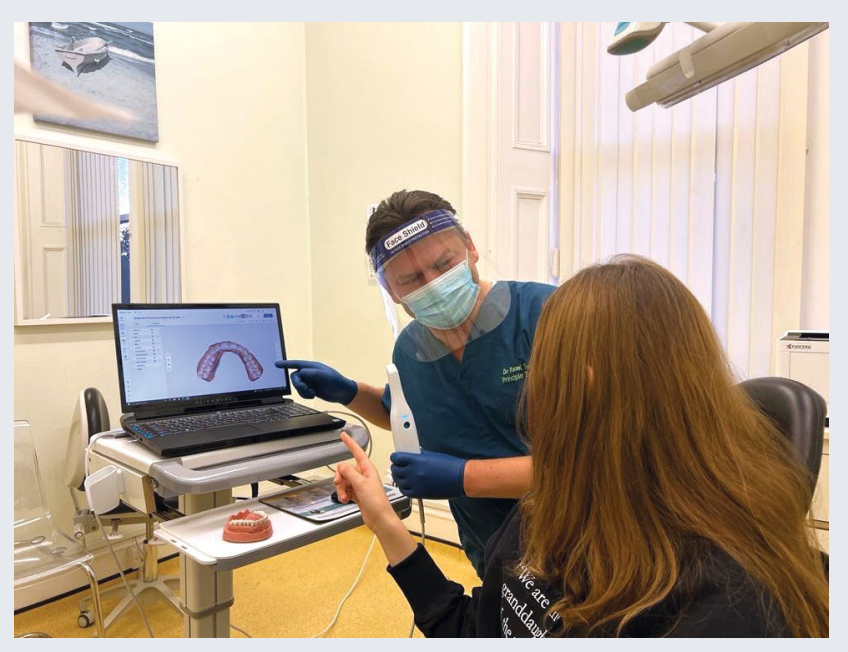

Two dental practices in Dundee, together with sister practices in Newburgh, Forfar and Kinross, may have become the most digitised dental surgeries in Scotland, with a $£ 250 \mathrm{k}$ investment bringing the very latest $3 \mathrm{D}$ digital intra oral scanning technology to patients in these areas.

Dr Rami Sarraf owns and runs First Alba Healthcare, which incorporates all five practices, with his wife, dentist Dr Ewa Plewa Sarraf. They have purchased the Medit i700 intra oral scanner across their five sites, a cutting edge scanner with powerful hardware and intelligent software, that will make the scanning experience a comfortable one for both dentist and patient.

The state-of-the-art equipment - used to create braces, retainers, bridges, crowns and other dental appliances - brings with it many significant benefits to patients, including a quicker, more comfortable experience when having appliances made, plus the opportunity for the patient to instantly see a full 3D image of their mouth on the monitor. This can lead to a greater understanding by the patient of what is being done to their teeth and gums, considerably aiding communication between them and the dentist in moving forward with future dental care.

Unlike most scanners in the market, the Medit i700 can be directly connected to the computer using a power delivery cable without a power hub and extra cables. This new feature of Medit i700 improves mobility and maintenance.

In terms of environmental impact, the new scanning technology carries with it a much lower carbon footprint, with appliances able to be manufactured on site, rather than transported back and forth to external labs. The amount of clinical waste generated in making appliances is also greatly reduced.

Having taken possession of the new equipment, Dr Sarraf is currently undertaking a mass staff training programme for key personnel so that they are ready to use the new equipment as soon as possible.

For more information about the Medit i700 visit https://i700. medit.com/.

\section{State-of-the-art guided bone regeneration material}

Tecnoss's OsteoBiol GTO is the state-of-the-art Second Generation Guided Bone Regeneration Material from Trycare.

All Tecnoss OsteoBiol products are Second Generation because they exhibit $100 \%$ conversion to human new bone within a finite, 12-24month period. This is unlike ceramatised first generation materials which only partially, if at all, convert to new bone, simply remaining as unreacted lumps of dead foreign bone within the GBR site.

Handling like a sticky putty, OsteoBiol GTO is Tecnoss's stateof-the-art ready-to-use pre-hydrated biomaterial that can be easily dispensed into defect sites direct from the syringe. It enables clinicians to skip the hydration phase with saline or blood, saving time and decreasing the risk of accidental exposure to pathogens. OsteoBiol GTO contains Tecnoss's innovative OsteoBiol TSV Gel which ensures optimal stickiness of the material, allowing easy adaptability to the recipient site and extreme stability.

Conceived as a universal biomaterial, easily adaptable to any bone defect, it is particularly indicated for horizontal augmentation of twowall defects and socket preservation with compromised buccal plates. It can also be used in sinus-lifting procedures where it can be applied directly through the bony window, helping to stabilise implants during immediate placement. It can also be used to treat peri-implant lesions and severe bony defects.

For more information, call Trycare Ltd on 01274885544 or visit www.trycare.co.uk/osteobiol.

\section{Software aims to halve the time spent on dental compliance}

Agilio Software, who provide technology solutions for the UK dental care sector, is launching a new and improved version of its compliance management tool, iComply NextGen, designed to retain its existing robustness and simplify the process to reduce the time dental practices spend on compliance by up to $50 \%$.

The launch follows significant investment by the healthcare technology group to advance its existing iComply system. It will introduce a new curation feature for their extensive compliance document library, based on priority levels.

iComply NextGen presents users with their relevant compliance literature, based on importance, assigning each task with a high, medium, or low priority level to reflect the statutory requirements and regulations. The new system has been designed to increase efficiency in the implementation of CQC and HIW regulations and readiness for inspections.

In the development process, the team at Agilio Software utilised their 20 years of experience in dental compliance and their thorough understanding of CQC and HIW demands, having helped dental practices across England and Wales to successfully pass over 1,000 inspections.

Agilio Software is one of the leading healthcare technology groups within the sector. As well as working in dental care, Agilio also operates in primary and secondary care.

Through its suite of software solutions, Agilio supports $78 \%$ of dental practices in the UK. For more information visit www. agiliosoftware.com. 